\title{
Significación pronóstica del PIN y la atípia glandular focal en la Biopsia Transrectal Ecodirigida de Próstata
}

\author{
Rodríguez-Patrón Rodríguez R, Mayayo Dehesa T, Burgos Revilla FJ*; Sanz Mayayo E, \\ García González R.
}

Unidad de Ecografia Urológica. *Universidad de Alcalá de Henares. Madrid.

Actas Urol Esp 2006; 30 (4): 359-366

\section{RESUMEN}

\section{SIGNIFICACIÓN PRONÓSTICA DEL PIN Y LA ATIPIA GLANDULAR FOCAL EN LA BIOPSIA TRANSRECTAL} ECODIRIGIDA DE PRÓSTATA

Objetivo: Revisar la incidencia de Neoplasia Intraepitelial Prostática (PIN) y Atípia Glandular Focal (AGF) en primera biopsia, el riesgo de estos pacientes de padecer cáncer en biopsias sucesivas y qué importancia se concede a estos hallazgos analizando con qué frecuencia son rebiopsiados estos pacientes y cuánto tiempo después del hallazgo inicial.

Método: Estudio retrospectivo sobre 6.000 pacientes biopsiados entre 1994 y 2002. No se han incluido pacientes con diagnóstico previo de cáncer. De los 6.000 pacientes 861 se han rebiopsiado en alguna ocasión sumando en conjunto 7.127 biopsias. Se ha realizado un estudio descriptivo que incluye los porcentajes y percentiles para variables cualitativas y medidas de tendencia central para las variables cuantitativas continuas.

Resultados: La incidencia de cáncer en la primera biopsia fué del 39,1\%. Las cifras de PIN o AGF se mantienen estables o aumentan progresivamente desde un 2 y $2,1 \%$ respectivamente en la primera biopsia hasta más del $6 \%$ en la cuarta y quinta biopsia. La media de tiempo entre biopsias en el caso de encontrar AGF o PIN en el diagnóstico inicial es de $180 \pm 221,6$ y $264 \pm 213,8$ días respectivamente. Sólo el 42 y el 40\% de pacientes con diagnóstico inicial de PIN o AGF se rebiopsian, encontrando finalmente un 45 y un $40 \%$ de tumores en las posteriores biopsias respectivamente.

Conclusión: La presencia de PIN o AGF implica un mayor riesgo de cáncer en biopsias posteriores; a pesar de ello menos de la mitad vuelven a biopsiarse. La realización de dos biopsias posteriores o una biopsia ampliada localiza la mayoría de los tumores asociados.

Palabras clave: Biopsia Transrectal de Próstata, PIN, Atípia Glandular Focal.

\section{ABSTRACT \\ PROGNOSTIC SIGNIFICANCE OF PIN AND ATYPICAL SMALL ACINAR PROLIFERATION ON TRANSRECTAL ULTRASOUND GUIDED PROSTATE BIOPSY}

Objective: To review the incidence of PIN and Atypical Small Acinar Proliferation (ASAP) on first biopsy, the risk to find cancer on following biopsies and what is the importance given to this findings, analizing how frequently and how long after the initial finding this patients are rebiopsied.

Method: We selected 6000 patients who underwent TRUS biopsy between 1994 and 2002. Patients with prior cancer diagnosis were not included. 861 of them underwent more than one biopsy, adding up to a total of 7127 biopsies. A descriptive study has been done including percentages and percentiles for qualitative variables, mean and median for continuous variables.

Results: Incidence of cancer on the first biopsy was 39,1\% .PIN and ASAP are stable or slightly increase from 2 and $2,1 \%$ respectively on the first biopsy to more than $6 \%$ on the fourth and fifth ones. Mean time between biopsies when ASAP or PIN are the initial findings is $180 \pm 221,6$ and $264 \pm 213,8$ days respectively. Just 42 and $40 \%$ of patients with prior PIN or ASAP diagnose are rebiopsied. On subsequent biopsies 45 and $40 \%$ of cancers were respectively found.

Conclusions: Presence of PIN or ASAP implies a higher cancer risk on subsequent biopsies; in spite of that, less than half of them are biopsied again. Performing two more biopsies or an amplified biopsy can find most of the tumors associated.

Keywords: Transrectal Ultrasound Guided Biopsy, PIN, ASAP. 
$\mathrm{E}^{\prime}$ objetivo de la Biopsia Transrectal Ecodirigida de Próstata (BTE) es confirmar o descartar la sospecha de Carcinoma Prostático (CP) en pacientes que presentan elevación de PSA o bien tacto rectal (TR) o Ecografía Transrectal (ETR) sospechosos. Cuando el diagnóstico anatomopatológico confirma la presencia de tumor o informa de la ausencia de malignidad, el significado queda claro. Sin embargo, existen dos entidades patológicas de significado más incierto, que sin implicar la existencia de malignidad, se asocian con una mayor posibilidad de ésta en caso de repetir la biopsia. Estas entidades son la Neoplasia Intraepitelial Prostática (PIN) y la Atípia Glandular Focal (AGF).

La PIN es el extremo preinvasivo del continuo de proliferación celular que tapiza las luces acinares y de los túbulos. Actualmente se emplea el acrónimo PIN para referirse al anterior PIN de alto grado que incluía los grados 2 y 3 de una escala de 1 a 3 , donde el PIN grado 1 por su variabilidad y falta de utilidad clínica ha perdido interés. En el resto del texto se empleará únicamente el término PIN. La OMS en su conferencia de consenso reconoce al PIN como la única lesión preinvasiva establecida para el cáncer de próstata $^{1}$.

Histológicamente el PIN se caracteriza por los mismos cambios citológicos que el carcinoma dentro de un acino o túbulo normal arquitecturalmente, incluyendo el agrandamiento nuclear y nucleolar. Existen 4 tipos de patrón:

1. en penacho, que es el más frecuente en las piezas de prostatectomía,

2. micropapilar,

3. plano y

4. cribiforme. En el $2 \%$ de los casos puede verse microinvasión estromal ${ }^{1,2}$.

La PIN no produce una imagen típica en ETR ni eleva por sí mismo las cifras de PSA pero su presencia se asocia a una incidencia de cáncer en sucesivas biopsias del 27 al 50\% $\%^{3,4}$.

La AGF refleja la presencia de cambios altamente sospechosos de malignidad, a pesar de los cuales el patólogo no puede confirmar el diagnóstico, lo que puede ser debido a una escasa representación de acini con los criterios citológicos y arquitecturales de carcinoma o cuando estos criterios no se encuentran presentes por completo $^{1}$. Para Bostwick ${ }^{1}$ no resulta posible el diagnóstico de certeza cuando existen menos de 3 acini sospechosos, salvo que se acompañen de una anaplasia citológica franca.

Otras entidades capaces de crear esta confusión son la adenosis, las lesiones tipo PIN, la atrofia no distinguible de cánceres atróficos, la inflamación asociada o los artefactos de inclusión ${ }^{2}$. Aunque la incidencia de AGF en biopsias es baja, se asocia a una incidencia de cáncer mayor del $50 \%$ en biopsias sucesivas ${ }^{1,4}$.

$\mathrm{Al}$ igual que con el PIN no existe una correlación característica con las imágenes ecográficas.

El objetivo de este trabajo es revisar en nuestra serie de BTE la incidencia de PIN y AGF en primera biopsia, el riesgo de estos pacientes de padecer cáncer en biopsias sucesivas y que importancia se concede a estos hallazgos, analizando con qué frecuencia son rebiopsiados estos pacientes y cuánto tiempo después del hallazgo inicial.

\section{MATERIAL Y MÉTODO}

Se han recogido los datos de 6.000 pacientes sometidos a BTE en los que se disponía de la anatomía patológica realizados desde final de 1994 hasta el año 2002. No se han incluido pacientes con diagnóstico previo de CP. De los 6.000 pacientes, 674 han sido biopsiados 2 veces, 128 tres veces, 43 cuatro veces, 13 en cinco ocasiones, 2 en seis y a un paciente se le practicaron 7 biopsias. En conjunto suponen 832 segundas, 185 terceras, 59 cuartas, 16 quintas, 3 sextas y una séptima biopsias, sumando en total 7.127 biopsias.

Todas las exploraciones han sido realizadas por los mismos miembros de la Unidad (TMD, RRP, ALZ) a pacientes remitidos desde diversos gabinetes urológicos privados.

Desde el inicio de la técnica de BTE en el año 1994, se han empleado 3 equipos de ecografía, todos pertenecientes a la línea Sonoline de Siemens Medical Systems, Inc. Ultrasound Group, Issaquah, WA. Para la obtención de muestras se utilizaron agujas 18 Gauge tipo Tru-Cut modificadas para ser disparadas mediante el dispositivo Biopty-Bard® (Bard Urological Inc., Atlanta, Georgia), que obtienen cilindros de tejido de 1,5-2 cm de longitud con un diámetro de aproximadamente $1 \mathrm{~mm}$. 
La preparación previa incluía como en muchos otros grupos la administración de antibiótico previo a la realización de la prueba, un enema comercial y una cápsula de metamizol magnésico.

De forma habitual se obtuvieron tres muestras de la parte más lateral de la zona periférica de cada lóbulo según se ha descrito previamente por nuestro grupo 5,6 . En caso de observarse zonas ecográficas sospechosas de tumor se incluyeron en las muestras del lóbulo correspondiente. De esta forma, el número de cilindros fue de seis de forma general, siendo excepción a esta regla únicamente aquellos pacientes con tumores avanzados, en los que el número de cilindros no modificaba la actitud terapéutica posterior o aquellos que por su situación médica o intolerancia a la prueba no se pudieron completar las muestras previstas.

La anatomía patológica se dividió en ausencia de malignidad (HPB por facilidad de nomenclatura), CP, PIN o AGF. Como se comentó en la introducción sólo se ha considerado el PIN de alto grado asimilando los casos de PIN de bajo grado, que en los primeros años aparecían, a la biopsia normal. Los criterios de PIN incluían los mismos cambios citológicos que el carcinoma dentro de un acino o túbulo normal arquitecturalmente, bajo el diagnóstico de AGF el patólogo agrupa aquellos cambios que le sugieren alta sospecha de malignidad pero sin ser conclusivos, bien por escasa representación de acinos patológicos, bien por otros motivos. En algunos pacientes coincidieron ambos diagnósticos. En éstos se han considerado incluido como AGF por ser el que mayor riesgo tiene de $\mathrm{CP}$ en sucesivas biopsias según la literatura.

Se ha realizado un estudio descriptivo que incluye los porcentajes para variables cualitativas (anatomía patológica) y medidas de tendencia central para las variables cuantitativas continuas (PSA, Densidad de PSA, relación PSA libre/PSA total, tiempo entre biopsias). Se ha realizado el análisis ANOVA para estudiar las diferencias de PSA, densidad de PSA, relación PSA libre/PSA total y volumen entre los diferentes grupos patológicos. El análisis se ha realizado con el paquete estadístico SPSS 11.0.

\section{RESULTADOS}

La incidencia general de CP considerando el total de biopsias en la población biopsiada fue del $42,6 \%$ (2.554/6.000). La incidencia de CP en la primera biopsia del 39,1\% (2.345/6.000). En las sucesivas biopsias el porcentaje de CP diagnosticados va disminuyendo a un 20,2\% (168/832), $16,8 \%$ (31/185) y 11,9\% (7/59) en las segunda, tercera y cuarta biopsias respectivamente. En la quinta biopsia repunta hasta el 18,8\% (3/16), aunque con un escaso número de pacientes. No aparecen tumores entre los pacientes con sexta o séptima biopsia (Fig. 1). Las biopsias sucesivas incrementan el diagnostico de $\mathrm{CP}$ en un $8,2 \%$ entre los pacientes con cáncer y un 3,5\% sobre el total de pacientes biopsiados.

Las cifras de PIN o AGF se mantienen estables o aumentan progresivamente desde un 2 y $2,1 \%$ respectivamente en la primera biopsia hasta más del 6\% en la cuarta y quinta biopsia. La Tabla 1 refleja los datos de anatomía patológica encontrados en las distintas biopsias.

Aunque el ANOVA intergrupos encuentra diferencias significativas en los valores de PSA, volumen, Densidad de PSA (DPSA) y relación entre PSA libre y total (PSA L/T) para las distintas anatomías patológicas, se aprecia que en pacientes con PIN o AGF, estas cifras se sitúan entre las de los pacientes con HPB y CP (Tabla 2).

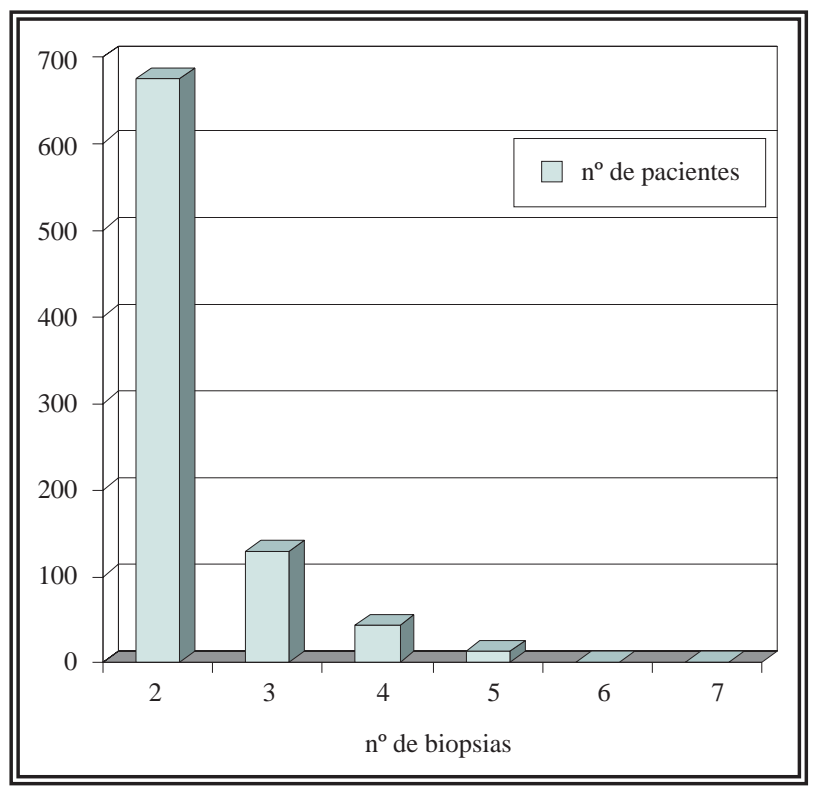

FIGURA 1. Distribución de pacientes rebiopsiados. 
Tabla 1

Frecuencias de anatomía patológica en sucesivas biopsias

\begin{tabular}{lccccc}
\hline $\begin{array}{l}\text { N(\%) } \\
\text { Nobiopsia }\end{array}$ & $\mathbf{N}$ & HPB (\%) & Cáncer (\%) & PIN(\%) & AGF(\%) \\
\hline $1^{\underline{a}}$ & 6000 & $3406(56,8)$ & $2345(39,1)$ & $122(2)$ & $127(2,1)$ \\
$2^{2^{\underline{a}}}$ & 832 & $611(73,4)$ & $168(20,2)$ & $25(3)$ & $28(3,4)$ \\
$3^{\underline{a}}$ & 185 & $143(77,3 \%)$ & $31(16,8)$ & $5(2,7)$ & $6(3,2)$ \\
$4^{\underline{a}}$ & 59 & $46(78)$ & $7(11,9)$ & $2(3,4)$ & $4(6,8)$ \\
$5^{\underline{a}}$ & 16 & $11(68,8 \%)$ & $3(18,8)$ & $1(6,3)$ & $1(6,3)$ \\
$6^{\underline{a}}$ & 3 & $2(66,7)$ & & $1(33,3)$ & \\
$7^{-a}$ & 1 & & & $1(100)$ & \\
\hline
\end{tabular}

La Tabla 3 refleja los hallazgos ecográficos y de TR en los diferentes grupos patológicos.

La Tabla 4 muestra el tiempo transcurrido entre las diferentes biopsias. Salvo en el caso del intervalo entre cuarta y quinta biopsia, en el resto supera de media el año, aunque varía de forma importante desde los 19 días hasta 6,36 años. La media de tiempo es menor en el caso de AGF o PIN en el diagnóstico inicial (180 221,6 y $264 \pm 213,8$ días respectivamente) o en la segunda biopsia $(305 \pm 277,2$ y $219 \pm 166,6$ dias respectivamente).
Del total de 6.000 pacientes, $122(2 \%)$ presentaban en la primera biopsia hallazgos compatibles con PIN. De ellos en 71 (58\%) no figuran biopsias posteriores, si bien puede que algunos de ellos se hayan biopsiado en otros centros o en fechas posteriores a la recogida de datos. Los restantes 51 pacientes (42\%) se sometieron a una segunda biopsia, 8 a una tercera, 4 y 2 pacientes a una cuarta y quinta biopsia respectivamente. En 23 de los 51 pacientes $(45 \%)$ se objetivó un tumor tras rebiopsiar en base a la existencia de PIN en la primera biopsia (Tabla 5).

En 127 de los 6.000 pacientes $(2,1 \%)$ se detectó inicialmente un AGF. De ellos en 50 (39,3\%) consta una segunda biopsia, en 6 una tercera y en 3 una cuarta. En total se han diagnosticado 20 tumores, lo que supone el 40\%. Otro 16\% continúa presentando PIN o AGF (Tabla 5).

\section{DISCUSIÓN}

La incidencia de PIN o de AGF de esta serie se encuentra en el rango de las publicaciones, aunque varía mucho en los distintos trabajos, osci-

Tabla 2

Descriptivos de PSA, volumen, DPSA y PSA L/T en primera biopsia por grupos de anatomía patológica

\begin{tabular}{|c|c|c|c|c|c|c|c|c|}
\hline & & $\mathbf{N}$ & Mediana & Media & DT & Min. & Máx. & ANOVA INTERGR.* \\
\hline \multirow{5}{*}{$\begin{array}{l}\mathrm{PSA} \\
\mathrm{ng} / \mathrm{ml}\end{array}$} & HBP & 3343 & 7.5 & 9.60 & 10.38 & .07 & 402.00 & \multirow[t]{5}{*}{$\mathrm{P}<0,0001$} \\
\hline & CANCER & 2307 & 10.6 & 42.32 & 193.81 & .09 & 4607.0 & \\
\hline & PIN & 121 & 8.2 & 10.86 & 11.14 & 1.44 & 94.28 & \\
\hline & AGF & 125 & 8.4 & 11.61 & 12.50 & 1.50 & 94.80 & \\
\hline & Total & 5896 & 8.35 & 22.47 & 122.53 & .07 & 4607.0 & \\
\hline \multirow{5}{*}{$\begin{array}{l}\text { VOLUMEN } \\
\text { c.c. }\end{array}$} & HBP & 3388 & 39. 71 & 46.45 & 85.71 & 3.53 & 483.7 & \multirow[t]{5}{*}{$\mathrm{P}<0,0001$} \\
\hline & CANCER & 2336 & 27.16 & 32.16 & 20.11 & 2.35 & 379.31 & \\
\hline & PIN & 122 & 39.62 & 42.19 & 17.93 & 13.44 & 112.69 & \\
\hline & $\mathrm{AGF}$ & 126 & 32.41 & 36.98 & 18.19 & 2.06 & 111.25 & \\
\hline & Total & 5972 & 34.68 & 40.57 & 66.23 & 2.06 & 483.7 & \\
\hline \multirow{5}{*}{$\begin{array}{l}\text { DPSA } \\
\mathrm{ng} / \mathrm{ml} / \text { c.c. }\end{array}$} & HBP & 3327 & 0.18 & .26 & .40 & .00 & 11.03 & \multirow[t]{5}{*}{$\mathrm{P}<0,0001$} \\
\hline & CANCER & 2298 & 0.41 & 1.39 & 5.44 & .01 & 88.76 & \\
\hline & PIN & 121 & 0.21 & .29 & .33 & .07 & 3.02 & \\
\hline & $\mathrm{AGF}$ & 124 & 0.23 & .33 & .30 & .05 & 2.13 & \\
\hline & Total & 5870 & 0.23 & .70 & 3.46 & .00 & 88.76 & \\
\hline \multirow[t]{5}{*}{$\mathrm{PSAL} / \mathrm{T}$} & HBP & 1401 & 0.15 & .16 & .07 & .01 & .63 & \multirow[t]{5}{*}{$\mathrm{P}<0,0001$} \\
\hline & CANCER & 831 & 0.10 & .12 & .06 & .00 & .43 & \\
\hline & PIN & 64 & 0.14 & .15 & .07 & .01 & .44 & \\
\hline & $\mathrm{AGF}$ & 49 & 0.13 & .14 & .07 & .03 & .39 & \\
\hline & Total & 2345 & 0.13 & .14 & .07 & .00 & .63 & \\
\hline
\end{tabular}

*En todas las variables la diferencia es significativa entre el grupo CP y el resto de diagnósticos anatomopatológicos. DT $=$ Desviación Típica 
Tabla 3

Anatomía patológica en función de los hallazgos del TR o la ETR en la primera biopsia.

\begin{tabular}{|c|c|c|c|c|c|c|}
\hline & & \multirow[b]{2}{*}{$\mathbf{n}$} & \multicolumn{4}{|c|}{ N (\%) } \\
\hline & & & HBP & CÁNCER & PIN & AGF \\
\hline \multirow{6}{*}{$\begin{array}{l}\text { Nódulo } \\
\text { hipoecoico } \\
\chi^{2} \mathrm{p}<0,0001\end{array}$} & NO & 4373 & 3041 & 1118 & 108 & 106 \\
\hline & & & $(69,5)$ & $(25,6)$ & $(2,5)$ & $(2,4)$ \\
\hline & SI & 1467 & 294 & 1147 & 11 & 15 \\
\hline & & & (20) & $(78,2)$ & $(0,7)$ & (1) \\
\hline & DUD & 139 & 58 & 73 & 3 & 5 \\
\hline & & & $(41,7)$ & $(52,5)$ & $(2,2)$ & $(3,6)$ \\
\hline \multirow{6}{*}{$\begin{array}{l}\text { Tacto rectal } \\
\chi^{2} p<0,0001\end{array}$} & NEG & 4667 & 3152 & 1288 & 109 & 118 \\
\hline & & & $(67,5)$ & $(27,6)$ & $(2,3)$ & $(2,5)$ \\
\hline & POS & 1163 & 183 & 961 & 11 & 8 \\
\hline & & & $(15,7)$ & $(82,6)$ & $(0,9)$ & $(0,7)$ \\
\hline & DUD & 85 & 25 & 58 & 1 & 1 \\
\hline & & & $(29,4)$ & $(68,2)$ & $(1,2)$ & $(1,2)$ \\
\hline \multirow{4}{*}{$\begin{array}{l}\text { Ecoestructura } \\
\chi^{2} p<0,0001\end{array}$} & HOM & 5133 & 3249 & 1649 & 117 & 118 \\
\hline & & & $(63,3)$ & $(32,1)$ & $(2,3)$ & $(2,3)$ \\
\hline & HET & 779 & 122 & 646 & 4 & 7 \\
\hline & & & $(15,7)$ & $(82,9)$ & $(0,5)$ & $(0,9)$ \\
\hline
\end{tabular}

$\mathrm{DUD}=$ dudoso; $\mathrm{NEG}=$ negativo; $\mathrm{POS}=$ positivo; $\mathrm{HOM}=$ homogénea; $\mathrm{HET}=$ heterogénea

Tabla 4

Tiempo transcurrido entre biopsias

\begin{tabular}{lcccccc}
\hline \multicolumn{7}{c}{ Tiempo transcurrido entre biopsias (en dias) } \\
& n & Media & Mediana & DT & Mínimo & Máximo \\
\hline$\Delta \mathrm{t} 2-1$ & 548 & 477,63 & 404 & 338,59 & 19 & 2322 \\
$\Delta \mathrm{t} 3-2$ & 149 & 431,41 & 377 & 258,96 & 27 & 1323 \\
$\Delta \mathrm{t} 4-3$ & 53 & 385,18 & 353 & 204,65 & 34 & 1134 \\
$\Delta \mathrm{t} 5-4$ & 15 & 371,60 & 341 & 180,79 & 121 & 809 \\
\hline
\end{tabular}

$\Delta \mathrm{t}=$ tiempo transcurrido entre las biopsias indicadas. DT = Desviación Típica

Tabla 5

Evolución de los pacientes con hallazgos de PIN o AGF como diagnóstico inicial.

\begin{tabular}{|c|c|c|c|c|c|c|}
\hline \multirow[b]{2}{*}{$\begin{array}{l}122 \text { pacientes } \\
\text { con PIN en }\end{array}$} & & \multicolumn{3}{|c|}{$\mathrm{N}(\%)$} & \multirow[b]{2}{*}{ PIN } & \multirow[b]{2}{*}{ AGF } \\
\hline & & $\mathbf{n}$ & HBP & CANCER & & \\
\hline \multirow[t]{4}{*}{$1^{\mathrm{a}}$ biopsia } & $2^{\mathrm{a}}$ biopsia & 51 & $26(51)$ & $18(35,3)$ & $6(11,8)$ & $1(2)$ \\
\hline & $3^{\text {a }}$ biopsia & 8 & $4(50)$ & $2(25)$ & $1(12,5)$ & $1(12,5)$ \\
\hline & $4^{\mathrm{a}} \stackrel{\text { biopsia }}{ }$ & 4 & $1(25)$ & $2(50)$ & & $1(25)$ \\
\hline & 5abiopsia & 2 & $1(50)$ & $1(50)$ & & \\
\hline
\end{tabular}

En total se diagnostican 23 tumores (45\% de los pacientes rebiopsiados)

\begin{tabular}{llccccc}
\hline 127 pacientes & & n & HBP & CANCER & PIN & AGF \\
con AGF en & $2^{\text {ab }}$ biopsia & 50 & $25(50)$ & $17(34)$ & $2(4)$ & $6(12)$ \\
$1^{\text {a }}$ biopsia & $3^{\text {abiopsia }}$ & 6 & $4(66,7)$ & $2(33,3)$ & & \\
& $4^{\text {ab }}$ biopsia & 3 & $2(66,7)$ & $1(33,3)$ & &
\end{tabular}

En total se diagnostican 20 tumores (40\% de los pacientes rebiopsiados) lando entre el 2 y el $12,9 \%$ para el primero $^{6-12}$ y el 2,5 y el $4,1 \%$ para las atipias ${ }^{7,11,12}$. Meng et al. ${ }^{13}$ en una revisión sobre datos de autopsia encuentran una incidencia de PIN del 25 al $43 \%$ en pacientes sin CP y del 63 al 94\% asociado a la presencia de $\mathrm{CP}$, lo que indica que al igual que ocurre con los tumores hallados en autopsia, la BTE subestima su presencia. Parece bastante establecido como se ha encontrado en esta serie, que el PIN no se asocia a mayores cifras de PSA, pues como indica Steiner $^{14}$ se trata de una lesión epitelial que no alcanza la basal y por tanto no entra en contacto con la vascularización. Los niveles de PSA algo mayores en pacientes con AGF probablemente se deban a la presencia de tumores no diagnosticados entre ellos.

La incidencia de PIN y AGF encontrada en la segunda biopsia también se sitúa en el rango encontrado en los trabajos revisados, de 3,2-6,9\% en el caso del PIN y de 0$8,7 \%$ para los $\mathrm{AGF}^{7,15-17}$.

Tanto los hallazgos de PIN y como los de AGF se relacionan con una probabilidad mayor de presentar cáncer en las siguientes biopsias, que varía según la literatura entre el 22 y el $51 \%^{11,14,18-21}$ y el 34 y el $51 \%^{17-19,22}$ respectivamente, comparativamente mayor que aquellos con biopsias normales. Este punto coincide con lo encontrado en esta serie, por lo que la presencia de estos hallazgos anatomopatológicos en una biopsia debe ser motivo suficiente para la realización de otra posterior, independientemente de las cifras de PSA que por otro lado muchos no encuentran relacionadas con la positividad en estos $\operatorname{casos}^{14,17,19,23}$.

De los 122 y 127 pacientes en los que se encontró PIN o AGF en la primera biopsia respectivamente, sólo 
51 y 50 en cada caso se realizaron al menos una segunda biopsia. Chan y Epstein ${ }^{17}$ refieren que menos del 64\% de sus pacientes con PIN fueron remitidos para rebiopsiar y en el caso de Renshaw et al. ${ }^{19}$ fueron 59 de 167 pacientes con AGF y 24 de 98 con PIN. Dada la alta frecuencia con que estos pacientes presentan $\mathrm{CP}$ en sucesivas biopsias es preocupante que un previsible $25 \%$ de los pacientes biopsiados inicialmente y con alguno de estos hallazgos puedan presentar un carcinoma y haberse perdido para el seguimiento, o bien no se han rebiopsiado por la normalización o estabilización de sus cifras de PSA, en cuyo caso iría a favor de que se tratase de tumores poco agresivos o significativos.

Puede observarse también que la incidencia de PIN o AGF en sucesivas biopsias es bastante estable o tiende al aumento, aunque sin significación estadística, mientras que desciende la posibilidad de CP. De hecho, en series de biopsias ampliadas, las cifras de PIN oscilan entre el 4,4 y el 4,8\% y las de AGF entre el 3,3 y el 4,8\% respectivamente ${ }^{24,25}$. Algo similar ocurre en la serie de nuestro grupo de BTE, con 10 muestras donde la incidencia de PIN es del 2,3\% y la de AGF del $4,5 \%$ (26). Esto podría reflejar en el caso del PIN la existencia de un grupo de pacientes en los que la única anomalía subyacente es el propio PIN que se van seleccionando según se retiran pacientes con tumor asociado. Puesto que la AGF indica únicamente la imposibilidad de diagnóstico de certeza, su estabilidad parece marcar un porcentaje de biopsias constante en el que no se podrá concretar el diagnóstico por diversos factores, igual que toda prueba tiene su porcentaje de falsos positivos y negativos.

El tiempo transcurrido entre las distintas biopsias supera de media el año, siendo máximo entre la primera y la segunda $(477,6 \pm 338,6$ días). En los casos en los que el diagnóstico patológico fue de PIN o de AGF, el tiempo transcurrido fue menor, aproximadamente 9 y 3 meses de media respectivamente. Para el AGF se encuentra en un margen razonable de tiempo pero ante el diagnóstico de PIN la rebiopsia se retrasa más y aunque esto no tenga probablemente influencia en la evolución posterior, caso de diagnosticarse finalmente un $\mathrm{CP}$, sí parece indicar que se atribuye una menor importancia al hallazgo de PIN que al de AGF. Ukimura et al. ${ }^{27}$ reflejan un tiempo medio entre las dos primeras biopsias del $446 \pm 389$ días y de $335 \pm 205$ entre segunda y tercera, muy similar a esta serie. En el ámbito espanol, Valer et al. ${ }^{28}$ muestran un tiempo medio de $91,7 \pm 133$ días y Gil Martínez et al. ${ }^{29}$ una mediana de 20 meses entre las dos primeras biopsias. Park et al. ${ }^{18}$ a diferencia de lo encontrado en este trabajo refieren un mayor tiempo entre las biopsias cuando el diagnóstico inicial fue de AGF $(23,8 \pm 6,6$ meses) que cuando fue de PIN $(10,6 \pm 4,2$ meses). Esta diferencia puede indicar la heterogeneidad de la importancia que otorgan a uno $\mathrm{u}$ otro hallazgo distintos urólogos.

La implicación del mayor riesgo asociado a estas alteraciones patológicas es la necesidad de biopsiar de nuevo. Lo que no queda igualmente claro es cuantas veces y cada cuanto tiempo parece razonable biopsiar para descartar un tumor asociado y significativo. Steiner et al. ${ }^{14}$ refieren que, por tratarse de una lesión premaligna que no puede monitorizarse mediante el PSA sérico, debe realizarse BTE cada 3-6 meses durante los dos primeros años y anualmente después. Parece excesivo este planteamiento, pues si bien es cierto que el PSA no se relaciona con su presencia, si lo hace con la del CP por lo que pacientes con PSA estable parecería poco probable que desarrollasen un tumor agresivo que pasara inadvertido. Lefkowitz et al. ${ }^{23}$ encuentran que de 72 pacientes que se rebiopsiaron tomando 12 cilindros por la presencia de un PIN previo con resultado negativo de tumor, 31 se sometieron 3 años después a una nueva biopsia encontrando un $25,8 \%$ de tumores en esta ocasión. Todos los que se sometieron a prostatectomía radical fueron localizados por lo que este tiempo de espera no influyó negativamente en la posibilidad de curación, concluyendo que pacientes con PIN, una vez rebiopsiados deben someterse a un nuevo procedimiento a los tres años independientemente de las cifras de PSA. Meng et al. ${ }^{13}$ aconsejan que si el diagnóstico de PIN o AGF ha sido por biopsia ampliada debe esperarse un año por la baja probabilidad de encontrar un tumor inmediatamente, pero en caso de biopsia sextante debe rebiopsiarse en los 3 a 6 meses siguientes, considerando la posibilidad de hacer una biopsia ampliada. Moore et al. ${ }^{30}$ apoyan este 
punto de vista al observar que únicamente 1 de 22 pacientes $(4,5 \%)$ con PIN en una primera biopsia ampliada presentaron tumor en la siguiente, no así aquellos con AGF, que a pesar del número inicial de muestras presentaron un 36\% de cánceres. Park et al. ${ }^{18}$ diagnostican el $91 \%$ y el $86 \%$ de los tumores asociados a PIN y a AGF respectivamente en la primera rebiopsia, teniendo en cuenta que se tomaron más de 6 muestras en el $64 \%$ de los pacientes. Bishara et al. ${ }^{31}$ identifican el $89,5 \%$ de los tumores presentes en pacientes con PIN como hallazgo de la biopsia inicial en las dos primeras biopsias, similar al 87\% encontrado en esta series, alcanzando el 95,7\% con tres. El 95\% de los tumores asociados a AGF en la primera biopsia precisó de dos biopsias posteriores para ser diagnosticados. Basados en estos hallazgos y en los datos de la literatura se considera prudente indicar la rebiopsia de 3 a 6 meses después del hallazgo inicial de PIN o AGF y completar hasta una tercera biopsia en caso de negatividad o bien plantear de inicio una segunda biopsia con 10-12 cilindros bajo anestesia local y vigilancia del PSA sérico y otros parámetros clínicos en caso de negatividad.

\section{CONCLUSIONES}

Tanto el PIN como el AGF son entidades anatomopatológicas que se relacionan con una mayor incidencia de CP en sucesivas biopsias.

Actualmente un número importante de pacientes con estos hallazgos no vuelven a biopsiarse con posterioridad.

Ante el hallazgo de PIN o AGF la realización de dos biopsias posteriores puede descartar razonablemente la presencia de CP asociado.

\section{REFERENCIAS}

1. Bostwick DG, Qian J, Schlesinger C. Contemporary pathology of prostate cancer. Urol Clin North Am 2003;30(2):181207.

2. Young RH, Srigley JR, Amin MB, Ulbright TM, Cubilla AL. Tumors of the prostate gland, seminal vesicles, male urethra, and penis. Atlas of tumor pathology. Armed forces institute of pathology. Washington D.C., 2000.

3. Epstein JL. Are you getting the maximum diagnostic and prognostic information from your prostate needle biopsies? Contemp Uro1 999;2:106-118.

4. Rodríguez-Patrón R, Mayayo T, González A, Lennie A, García R, Cuesta C. ¿Pueden los índices basados en el PSA determinar en qué pacientes se debe repetir la biopsia transrectal ecodirigida prostática? Estudio sobre 546 pacientes rebiopsiados. Arch Esp Urol 2002;55:1225-1234.
5. Rodríguez R, Mayayo T, Galbis F, Jiménez M, Burgos FJ, Allona A et al. Utilidad clínica de las pruebas diagnósticas disponibles en el carcinoma prostático. Resultados sobre 500 biopsias. Parte I: PSA, densidad de PSA y PSA predecible. Arch Esp Urol 1997;50:333-338.

6. Mayayo T, Rodríguez-Patrón R, Lennie A, Arias F, Carrera C, García R. Biopsia transrectal ecodirigida. Análisis de los resultados de una serie de 1900 pacientes. Arch Esp Urol 1999;52:453-463.

7. O'dowd GJ, Miller MC, Orozco R, Veltri RW. Analysis of repeated biopsy results within 1 year after a noncancer diagnosis. Urology 2000;55(4):553-559.

8. Orozco R, O'dowd GJ, Kunnel B, Miller MC, Veltri RW. Observations on pathology trends in 62,537 prostate biopsies obtained from urology private practices in the United States. Urology 1998;51(3):186-195.

9. Roehl KA, Antenor JA, Catalona WJ. Serial biopsy results in prostate cancer screening study. J Urol 2002;167(6):24352439.

10. Frauscher F, Klauser A, Volgger H, Halpern EJ, Pallwein L, Steiner $\mathrm{H}$ et al. Comparison of contrast enhanced color doppler targeted biopsy with conventional systematic biopsy: impact on prostate cancer detection. J Urol 2002; 167(4):1648-1652.

11. Wills ML, Hamper UM, Partin AW, Epstein JI. Incidence of high-grade prostatic intraepithelial neoplasia in sextant needle biopsy specimens. Urology 1997;49(3):367-373.

12. Morote Robles J, López López M, Encabo Duro G, De Torres Ramírez I. Incidencia de las lesiones asociadas en la biopsia prostática y su influencia en la concentración sérica del antígeno prostático específico. Actas Urol Esp 1999; 23(6):400-405.

13. Meng MV, Shinohara K, Grossfeld GD. Significance of high-grade prostatic intraepithelial neoplasia on prostate biopsy. Urol Oncol 2003;21(2):145-151.

14. Steiner MS. High grade prostatic intraepithelial neoplasia is a disease. Curr Urol Rep 2001;2:195-198.

15. Fleshner NE, O'sullivan M, Fair WR. Prevalence and predictors of a positive repeat transrectal ultrasound guided needle biopsy of the prostate. J Urol 1997;158:505-508.

16. García González R, Mayayo T, Lennie A, Rodríguez-Patrón $\mathrm{R}$, Cuesta C. Repetición de la biopsia prostática ecodirigida para la detección de cáncer. Estudio de una serie de 192 pacientes re-biosiados. Actas Urol Esp 2000;24(8): 644-651.

17. Chan TY, Epstein JI. Follow-up of atypical prostate needle biopsies suspicious for cancer. Urology 1999;53:351-355.

18. Park S, Shinohara K, Grossfeld GD, Carroll PR. Prostate cancer detection in men with prior high grade prostatic intraepithelial neoplasia or atypical prostate biopsy. J Urol 2001;165(5): 1409-1414.

19. Renshaw AA, Santis WF, Richie JP. Clinicopathological characteristics of prostatic adenocarcinoma in men with atypical prostate needle biopsies. J Urol 1998;159(6): 2018-2021.

20. Weinstein MH, Epstein JI. Significance of high-grade prostatic intraepithelial neoplasia on needle biopsy. Hum Pathol 1993;24(6):624-629.

21. Kamoi K, Troncoso P, Babaian RJ. Strategy for repeat biopsy in patients with high grade prostatic intraepithelial neoplasia. J Urol 2000;163(3):819-823.

22. Allen EA, Kahane H, Epstein JI. Repeat biopsy strategies for men with atypical diagnoses on initial prostate needle biopsy. Urology 1998;52(5):803-807. 
23. Lefkowitz GK, Taneja SS, Brown J, Melamed J, Lepor H. Follow-up interval prostate biopsy 3 years after diagnosis of high grade prostatic intraepithelial neoplasia is associated with high likelihood of prostate cancer, independent of change in prostate specific antigen levels. J Urol 2002;168 (4 Pt1):1415-1418.

24. Presti JC Jr, O’dowd GJ, Miller MC, Mattu R, Veltri RW. Extended peripheral zone biopsy schemes increase cancer detection rates and minimize variance in prostate specific antigen and age related cancer rates: results of a community multi-practice study. J Urol 2003;169(1):125-129.

25. Rodríguez Duarte C. Biopsia prostática multifragmentaria. Arch Esp Urol 2002;55:907-914.

26. Rodríguez-Patrón R, Mayayo T, Alonso M, Burgos FJ, García R, Lennie A. Rentabilidad de la biopsia ampliada con 10 muestras prostáticas. Estudio prospectivo sobre 222 pacientes. Arch Esp Urol (en prensa).

27. Ukimura O, Durrani O, Babaian RJ. Role of PSA and its indices in determining the need for repeat prostate biopsies. Urology 1997;50(1):66-72.

28. Valer J, Agüera L, Adot JM, Virseda M, Marcos J, Gómez A et al. Parámetros clínicos y analíticos que indicarían la realización de una segunda biopsia en pacientes con PSA elevado y biopsia previa negativa. Actas Urol Esp 2002; 26(3):196-203.

29. Gil Martínez P, Allepuz C, Gil MJ, Oliva J, Andrés V, Valdivia $\mathrm{P}$ et al. Rebiopsia de próstata. factores pronósticos del resultado anatomopatológico. Actas Urol Esp 2000;25(5):560-567.

30. Moore CK. Karikehalli S. Nazeer T. Fisher HA. Kaufman RP Jr. Mian BM. Prognostic significance of high grade prostatic intraepithelial neoplasia and atypical small acinar proliferation in the contemporary era. J Urol 2005;173(1):70-72.

31. Bishara T, Ramnani D, Epstein JI. High grade Prostatic Intraepithelial Neoplasia on needle biopsy. Risk of cancer on repeat biopsy related to number of involved cores and morphologic pattern. Am J Surg Pathol 2004;28(5):629-633.

Dr. R. Rodríguez-Patrón

E-mail: rafael.rodriguezpatron@wanadoo.es

(Trabajo recibido el 2 de noviembre 2005) 